\title{
Fuzzy modelling of visual texture: coarseness, contrast and directionality properties
}

\author{
P.M. Martínez-Jiménez ${ }^{1}$ J. Chamorro-Martínez ${ }^{1}$ J.M. Soto-Hidalgo ${ }^{2}$ \\ ${ }^{1}$ Department of Computer Science and Artificial Intelligence, University of Granada, Spain \\ ${ }^{2}$ Department of Computer Architecture, Electronics and Electronic Technology, Univ. of Córdoba, Spain
}

\begin{abstract}
The analysis of the perceptual properties of texture plays a fundamental role in tasks like semantic description of images or content-based image retrieval using linguistic queries. In this paper, we propose to model these properties by means of fuzzy sets defined on the domain of some representative measures. In our approach, the membership functions associated to these fuzzy sets are obtained by learning a functional relationship between the computational values given by the measures and the human perception of the properties. The performance of each fuzzy set is analyzed and tested with human assessments, allowing us to identify the most suitable model to represent each property.
\end{abstract}

Keywords: Image features, texture modelling, fuzzy sets, human perception

\section{Introduction}

The most natural way for humans to describe visual texture is by using some perceptual properties, like coarseness, directionality, contrast, line-likeness or regularity $[1,2]$. The analysis of these properties in images can be very useful in fields where some interaction with subjects is needed. For example, it plays a fundamental role in tasks like semantic description of images [3, 4, 5] or in content-based image retrieval systems using linguistic queries $[6$, $7,8]$, where a perceptual texture characterization is needed.

There are many measures in the literature that, given an image, capture the presence of these texture properties in the sense that the greater the value given by the measure, the greater (lower) the presence of the property $[9,10]$. However, there is no perceptual relationship between the value given by these measures and the degree in which the humans perceive the texture. For example, given a certain value calculated by applying a coarseness measure to an image, there is not an immediate way to decide whether there is a coarse texture, a fine texture or something intermediate (i.e. there is not a textural interpretation).

The imprecision associated to these measures suggests the use of representation models that incorporate the uncertainty. Nevertheless, the majority of the approaches that can be found in the literature are crisp proposals $[11,9,12]$ which do not model any kind of imprecision. To face this problem, some proposals arise from the fuzzy set field, and more specifically from the content-based image retrieval area $[6,8,13,14]$. In these proposals, a mapping from low-level statistical features (the crisp measures described above) to high level textural concepts is performed by defining membership functions for each textural feature.

However, in all these fuzzy approaches the membership functions are adjusted manually or by using a fuzzy clustering, but without considering the relationship between the measure values and the human perception of the property. This implies that the obtained membership degrees do not necessarily match what a human would expect. In addition, all these fuzzy approaches do not propose a global modelling of the textural concept, but a fuzzy partition providing a set of linguistic terms associated to this concept. This type of solution is unsuitable for some classical tasks, like pattern recognition, because a single presence degree of the textural property cannot be obtained, but one membership degree for each linguistic term in the partition.

In this paper, we propose a perception-based fuzzy approach for texture modelling in order to solve all these problems. In this approach, each texture property is modelled by means of a unique fuzzy set defined on the domain of a representative crisp measure. Thus, the obtained fuzzy set will directly represent the presence degree of the property, allowing its use in pattern recognition problems, as it will be shown in section 5 . In order to obtain the membership function, a functional relationship between the computational values given by the measures and the human perception of the corresponding property is learned. This way, the presence degree given by the obtained fuzzy set will match what a human would expect. According to the psychological experiments performed by Tamura et al. in [2], coarseness, contrast and directionality are considered the three most important texture properties, playing a fundamental role in human visual interpretation $[8,15,16]$. In this paper, we will focus our study on these properties.

The rest of the paper is organized as follows. In section 2 a general overview introducing our methodology is presented. After that, some ele- 
ments of the model are described in detail in the following sections; concretely, the way to obtain human assessments about the perception of the properties is faced in section 3 , while section 4 describes the method employed to obtain the membership functions of the proposed fuzzy sets. In section 5 some results obtained by applying the models are shown, and the main conclusions are summarized in section 6 .

\section{Fuzzy modelling of texture properties: an overview of the proposal}

As mentioned in the above section, in this paper we propose to model texture properties by means of fuzzy sets. From now on, let $\mathcal{P}=\left\{\right.$ fineness $^{1}$, contrast, directionality\} be the set of texture properties that will be modelled in this paper and let $\mathcal{F}^{p}=\left\{F_{1}^{p}, \ldots, F_{K_{p}}^{p}\right\}$ be a set of representative computational measures of the property $p \in \mathcal{P}$. In our approach, we propose to model a texture property $p \in \mathcal{P}$ as a fuzzy set $\mathcal{T}_{k}^{p}$ defined on the domain of a measure $F_{k}^{p}$, where the membership function ${ }^{2}$ of this fuzzy set will be defined as

$$
\mathcal{T}_{k}^{p}: \mathbb{R} \rightarrow[0,1]
$$

For this modelling, two questions need to be faced: (i) what reference set should be used for the fuzzy set, and (ii) how to obtain the related membership function. Regarding the reference set, we will define the fuzzy set on the domain of a given computational measure $F_{k}^{p} \in \mathcal{F}^{p}$. The measures analyzed in this paper, corresponding to fineness, contrast and directionality properties, are listed in the first column of Table 1. It should be noticed that most of these measures were developed in the early nineties. However, they are still considered the state of the art in the characterization of the perceptual properties of texture. All of them are automatically computed from the texture images.

Regarding the membership function, we propose to obtain it by using a perceptually-based approach that relates the computational measures with the human perception of the property. For this purpose, two questions need to be faced: firstly, how to obtain the data about the "human perception" of the property and, secondly, how to fit these data with the measures in order to obtain the membership function. To get information about the human perception of a texture property $p \in \mathcal{P}$, a set of images covering different presence degrees of this property will be gathered. These images will be used to collect, by means of a poll, human assessments about the perceived presence of the property. From

\footnotetext{
${ }^{1}$ Let us remark that "coarseness" and "fineness" are opposite but related textural concepts. The advantage of modelling the concept of fineness is that the maximum presence of this property in the image is delimited by the size of pixel.

${ }^{2}$ To simplify the notation, as it is usual in the scope of fuzzy sets, we will use the same notation $\mathcal{T}_{k}^{p}$ for the fuzzy set and for the membership function that defines it.
}

now on, let $\mathcal{I}^{p}=\left\{I_{1}^{p}, \ldots, I_{N_{p}}^{p}\right\}$ be the set of $N_{p}$ images representing examples of the property $p \in \mathcal{P}$, and let $\Gamma^{p}=\left\{v_{1}^{p}, \ldots, v_{N_{p}}^{p}\right\}$ be the set of values associated to $\mathcal{I}^{p}$, with $v_{i}^{p}$ being the value representing the presence degree of the property $p \in \mathcal{P}$ perceived by humans in the image $I_{i}^{p} \in \mathcal{I}^{p}$. The description of the texture image set and the way to obtain $\Gamma^{p}$ are detailed in section 3 .

To obtain the membership function $\mathcal{T}_{k}^{p}$ for a given measure $F_{k}^{p} \in \mathcal{F}^{p}$ of the texture property $p \in \mathcal{P}$, a robust fitting method will be applied in order to obtain suitable functions relating the values of the measure calculated for each image with the presence degree of the property $p$ perceived by humans. This fitting method is described in section 4 .

\section{Assessment Collection}

In this section, the way to obtain the set of values $\Gamma^{p}=\left\{v_{1}^{p}, \ldots, v_{N_{p}}^{p}\right\}$, that represent the presence degree of the property $p \in \mathcal{P}$ perceived by humans in the images $I_{i}^{p} \in \mathcal{I}^{p}$, will be described. For this purpose, firstly the image set $\mathcal{I}^{p}$ will be selected (section 3.1). After that, a poll for getting assessments about the perception of the property will be designed (section 3.2). Finally, for a given image, the assessments of the different subjects will be aggregated (3.3).

\subsection{The texture image set}

For each property $p \in \mathcal{P}$, a set $\mathcal{I}^{p}=\left\{I_{1}^{p}, \ldots, I_{N_{p}}^{p}\right\}$ of $N_{p}=80$ images representing examples of this property has been selected. Figure 1 shows some images extracted from the set $\mathcal{I}^{p}$ corresponding to the properties of fineness (Figure 1(a)), contrast (Figure 1(b)) and directionality (Figure 1(c)). Each set has been selected satisfying the following conditions:

- It covers the different presence degrees of the property.

- The number of images for each presence degree is representative enough.

- Each image shows, as far as possible, just one presence degree of the property.

Due to the third condition, each image can be viewed as "homogeneous" respect to the presence degree of the corresponding property, i.e., if we select two random windows (with a dimension which does not "break" the original texture primitives and structure), the perceived presence of the property will be the same for each window (and also with respect to the original image). In other words, we can see each image $I_{i}^{p} \in \mathcal{I}^{p}$ as a set of lower dimension images (sub-images) with the same presence degree of the original one. This will be very useful for the fitting process, because we can have a larger number of fitting points without extending the number of images used in the poll. 


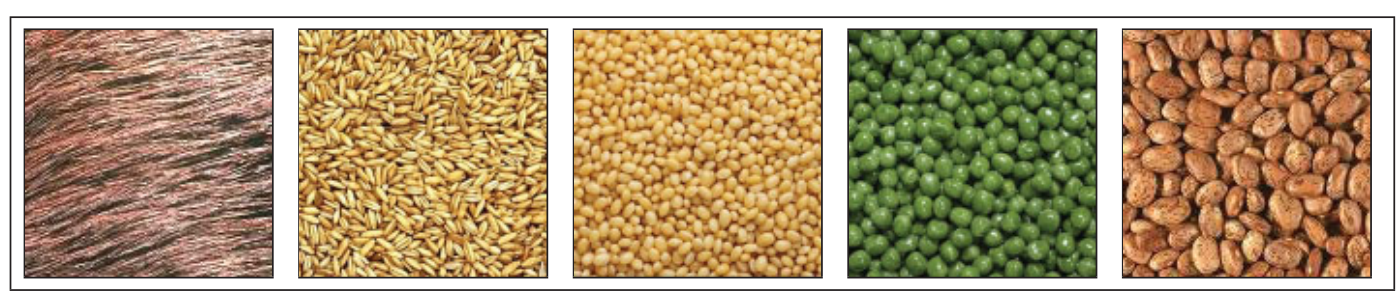

(a)

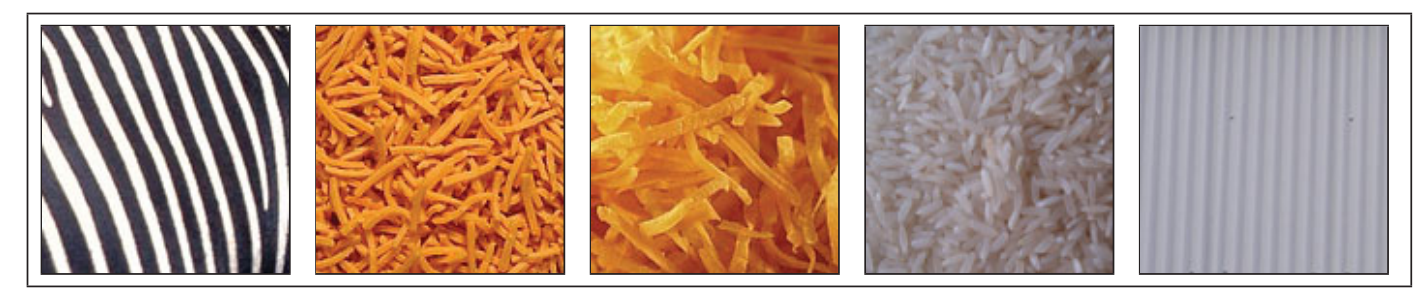

(b)


(c)

Figure 1: Examples of the texture images used in the poll corresponding to the properties of fineness (a), contrast (b) and directionality (c).

\subsection{The poll}

Given the image set $\mathcal{I}^{p}$, the next step is to obtain assessments about the perception of the corresponding property $p \in \mathcal{P}$ from a set of subjects. From now on, we shall note as $\Theta^{p, i}=\left[o_{1}^{p, i}, \ldots, o_{L}^{p, i}\right]$ the vector of assessments obtained from $L$ subjects for the image $I_{i}^{p}$. We considered three alternatives to get $\Theta^{p, i}:$

- To ask subjects about a presence degree between 0 and 1 for each image in the set.

- To ask subjects to describe the image property using linguistic labels in a pre-defined term set (e.g., very low, medium, high, etc.), so that each label has associated a presence degree.

- To ask subjects to describe the image property using linguistic labels, where each label has associated a visual example that represents the corresponding presence degree.

The first choice allows subjects to have more freedom to assess the presence degree of the property. However, according to our own experience, it is very difficult for a subject to provide a value between 0 and 1 that represents the presence degree of a certain texture concept (except in the case of both extremes: fulfillment of the concept -degree of 1- and unfulfillment of the concept -degree of 0). Thus, this alternative was discarded.
The above problem is solved by the second choice. The subject does not assess a value about the presence degree of the texture property, but gives his opinion using a set of linguistic labels related to the corresponding property. Each linguistic term has an associated presence degree (distributed gradually in $[0,1]$ ), so the requested assessments can be directly obtained. Note that this solution is equivalent to assign images to classes, where each class has associated a linguistic term (and therefore, a presence degree). The main drawback of this approach is that only a few linguistic labels can be considered for each property, as it is very dificult for humans to discriminate between more classes without using visual examples associated to them.

This problem is solved by the third choice. This solution is an extension of the previous one, in which each linguistic label has an associated visual example that represent the corresponding presence degree. Thus, humans are able to discriminate between more different classes. From now on, let $R^{p}$ be the number of classes that have been considered in the poll for the property $p \in \mathcal{P}$. One of the classes represents the presence degree of 1 of this property. In our proposal, traditional examples used in the literature to define very fine, high contrasted and very directional textures has been considered for this class [17]. An example that represents this presence degree for each property is shown in the first image of figures $1(\mathrm{a}), 1(\mathrm{~b})$ and $1(\mathrm{c})$, respec- 
tively. Another of the classes considered in the poll represents the presence degree of 0 of the property. In this case, again, traditional examples used in the literature to define very coarse, very low contrasted and very non-directional textures has been considered for this class. The last image of figures 1(a) and 1(b) shows an example of this presence degree for fineness and contrast properties, respectively. In the case of directionality, the presence degree of 0 is associated to texture primitives that do not have a dominant dimension or their arrangement does not have a dominant orientation, as it is shown in the last two images of Figure 1(c).

The rest of classes represent presence degrees of the property between 0 and 1 . In the case of fineness, nine classes have been regarded, considering gradual variations in the size of texture primitives, as it is shown in the sample images of Figure 1(a). It should be noticed that these images are in decreasing order according to the presence degree of the fineness concept. In the case of contrast, five classes have been considered, taking into account gray level differences in texel edges in order to scale their contrast between the classes corresponding to degrees 1 and 0 . The five representative images used in the poll are shown in Figure 1(b). In the case of directionality, six classes have been regarded, considering gradual variations in texels orientation or/and shape, as it is shown in the second and third images of Figure 1(c).

In our approach, 20 subjects have participated in the poll. As result, a vector of 20 assessments $\Theta^{p, i}=$ $\left[o_{1}^{p, i}, \ldots, o_{20}^{p, i}\right]$ is obtained for each image $I_{i}^{p} \in \mathcal{I}^{p}$. The degree $o_{j}^{p, i} \in[0,1]$ associated to the assessment given by the subject $S_{j}$ to the image $I_{i}^{p}$ is computed as $o_{j}^{p, i}=\left(R^{p}-k\right) /\left(R^{p}-1\right)$, where $k \in\left\{1, \ldots, R^{p}\right\}$ is the index of the class to which the image is assigned by the subject.

\subsection{Assessment aggregation}

Our aim at this point is to obtain, for each image in the set $\mathcal{I}^{p}$, one assessment $v_{i}^{p}$ that summarizes the assessments $\Theta^{p, i}$ given by the different subjects about the presence degree of the property $p \in \mathcal{P}$. To aggregate opinions we have used an OWA operator guided by a quantifier [18]. Concretely, the quantifier "the most" has been employed, which allows to represent the opinion of the majority of the subjects. This quantifier is defined as

$$
Q(r)=\left\{\begin{array}{lr}
0 & \text { if } r<a \\
\frac{r-a}{b-a} & \text { if } a \leq r \leq b \\
1 & \text { if } r>b
\end{array}\right.
$$

with $r \in[0,1], a=0.3$ and $b=0.8$. Once the quantifier $Q$ has been chosen, the weighting vector of the OWA operator can be obtained following [18] as $w_{j}=Q(j / L)-Q((j-1) / L), j=1,2, \ldots, L$. According to this, for each image $I_{i}^{p} \in \mathcal{I}^{p}$, the vector
Table 1: Fitting errors and test errors related to each measure for the properties of fineness (a), contrast (b) and directionality (c).

\begin{tabular}{|l|c|c|}
\hline $\begin{array}{l}\text { Fineness } \\
\text { measure }\end{array}$ & $\begin{array}{c}\text { Fitting } \\
\text { error }\end{array}$ & $\begin{array}{c}\text { Test } \\
\text { error }\end{array}$ \\
\hline Amadasun [10] & 0.1333 & 0.1695 \\
Correlation [9] & 0.1401 & 0.1747 \\
Abbadeni [19] & 0.1639 & 0.1947 \\
FD [20] & 0.1776 & 0.2084 \\
Tamura [2] & 0.1913 & 0.2070 \\
ED [21] & 0.2009 & 0.2168 \\
DGD [22] & 0.2031 & 0.2339 \\
LH [9] & 0.2150 & 0.2336 \\
Weszka [23] & 0.2157 & 0.2411 \\
Contrast [9] & 0.2161 & 0.2475 \\
SNE [24] & 0.2267 & 0.2418 \\
SRE [25] & 0.2296 & 0.2455 \\
\hline
\end{tabular}

(a)

\begin{tabular}{|l|c|c|}
\hline $\begin{array}{l}\text { Contrast } \\
\text { measure }\end{array}$ & $\begin{array}{c}\text { Fitting } \\
\text { error }\end{array}$ & $\begin{array}{c}\text { Test } \\
\text { error }\end{array}$ \\
\hline Tamura [2] & 0.0340 & 0.0649 \\
Amadasun [10] & 0.0780 & 0.1108 \\
Abbadeni [19] & 0.1003 & 0.1393 \\
Haralick [9] & 0.1157 & 0.1416 \\
\hline
\end{tabular}

(b)

\begin{tabular}{|l|c|c|}
\hline $\begin{array}{l}\text { Directionality } \\
\text { measure }\end{array}$ & $\begin{array}{c}\text { Fitting } \\
\text { error }\end{array}$ & $\begin{array}{c}\text { Test } \\
\text { error }\end{array}$ \\
\hline Tamura [2] & 0.0687 & 0.1064 \\
Abbadeni [19] & 0.0788 & 0.1324 \\
Fourier [23, 26] & 0.1073 & 0.1406 \\
\hline
\end{tabular}

(c)

$\Theta^{p, i}$ obtained from $L$ subjects will be aggregated into one assessment $v_{i}^{p}$ as follows:

$$
v_{i}^{p}=w_{1} \hat{o}_{1}^{p, i}+w_{2} \hat{o}_{2}^{p, i}+\ldots+w_{L} \hat{o}_{L}^{p, i}
$$

where $\left[\hat{o}_{1}^{p, i}, \ldots, \hat{o}_{L}^{p, i}\right]$ is a vector obtained by ranking in nonincreasing order the values of the vector $\Theta^{p, i}$.

\section{Fitting the membership function}

At this point, the aim is to obtain, for a given measure $F_{k}^{p} \in \mathcal{F}^{p}$ of a property $p \in \mathcal{P}$, the corresponding membership function $\mathcal{T}_{k}^{p}$. In this paper, we propose to find a function that associates the measure values of the property with the corresponding human assessments about it. As it was pointed out in section 3.1, thanks to the "homogeneity" in the presence degree of the property, each image $I_{i}^{p} \in \mathcal{I}^{p}$ can be seen as a set of sub-images with the same presence degree $v_{i}^{p}$ of the original one. From now on, we will note as $\mathcal{I}_{\mathcal{W}}^{p}=\left\{I_{i, w}^{p}, i=1, \ldots, N_{p} ; w=\right.$ $1, \ldots, W\}$ the set of sub-images extracted from $\mathcal{I}^{p}$, 
where $I_{i, w}^{p}$ is the $w$-th sub-image of $I_{i}^{p}$ and $W$ is the number of sub-images considered for each image; on the other hand we will note as $m_{i, w}^{p, k}$ the result of applying the measure $F_{k}^{p}$ to the sub-image $I_{i, w}^{p}$. According to this notation, let $\mathcal{I}_{\mathcal{W} \text {, fit }}^{p} \subseteq \mathcal{I}_{\mathcal{W}}^{p}$ and $\mathcal{I}_{\mathcal{W}, \text { test }}^{p}=\mathcal{I}_{\mathcal{W}}^{p} \backslash \mathcal{I}_{\mathcal{W}, \text { fit }}^{p}$ be two complementary subsets of $\mathcal{I}_{\mathcal{W}}^{p}$, that will be used for fitting the membership function and testing the obtained model, respectively.

Thus, in order to estimate the membership function that associates the measure values $\left(m_{i, w}^{p, k}\right)$ and the human assessments $\left(v_{i}^{p}\right)$, we propose to fit a suitable curve to the subset of points:

$$
\Psi_{\text {fit }}^{p, k}=\left\{\left(m_{i, w}^{p, k}, v_{i}^{p}\right) ; I_{i, w}^{p} \in \mathcal{I}_{\mathcal{W}, f i t}^{p}\right\}
$$

In this paper, for each image $I_{i}^{p} \in \mathcal{I}^{p}, W=200$ sub-images of size $32 \times 32$ have been considered, so $\mathcal{I}_{\mathcal{W}}^{p}$ is formed by 16000 sub-images for each property $p \in \mathcal{P}$. We propose to randomly select $75 \%$ of them for the fitting, so 12000 points are contained within $\Psi_{f i t}^{p, k}$.

The measure values can be affected by some factors, like brightness, contrast or noise, which typically causes outliers in the fitting points. For this reason, in our approach the membership function is calculated by means of a Robust Fitting of the set $\Psi_{f i t}^{p, k}$. In this modelling, the robust fitting based on M-estimators (a generalization of the least squares fitting) is used [27]. In addition, to define $\mathcal{T}_{k}^{p}$, the following considerations are taken into account:

- $\mathcal{T}_{k}^{p}$ should be a monotonic function.

- The values $\mathcal{T}_{k}^{p}(x)=0$ and $\mathcal{T}_{k}^{p}(x)=1$ should be achieved from a certain value.

Regarding the above properties, we propose to define $\mathcal{T}_{k}^{p}$ as a function of the form ${ }^{3}$

$\mathcal{T}_{k}^{p}\left(x ; a_{n} \ldots a_{0}, \alpha, \beta\right)= \begin{cases}1 & x<\alpha, \\ p o l y\left(x ; a_{n} \ldots a_{0}\right) & \alpha \leq x \leq \beta, \\ 0 & x>\beta\end{cases}$

with $\operatorname{poly}\left(x ; a_{n} \ldots a_{0}\right)$ being a polynomial function

$$
\operatorname{poly}\left(x ; a_{n} \ldots a_{0}\right)=a_{n} x^{n}+\ldots+a_{1} x^{1}+a_{0}
$$

In our proposal, the parameters $a_{n} \ldots a_{0}, \alpha$ and $\beta$ of the function $\mathcal{T}_{k}^{p}$ are calculated by carrying out a robust fitting on $\Psi_{f i t}^{p, k}$. For the polynomial function, the cases of $n=1,2,3$ (i.e. linear, quadratic and cubic functions) have been considered, with the constraint to obtain a strictly monotonic function between $\alpha$ and $\beta$.

The second column of tables 1(a), 1(b), and 1(c) show the least fitting error related to each measure

\footnotetext{
${ }^{3}$ Note that this function is defined for measures that decrease according to the perception of fineness. For those that increase, the function needs to be changed appropriately, i.e. it takes the value 0 for $x<\beta$, it takes the value 1 for $x>\alpha$, and the polynomial function is computed for $\beta \leq x \leq \alpha$.
}
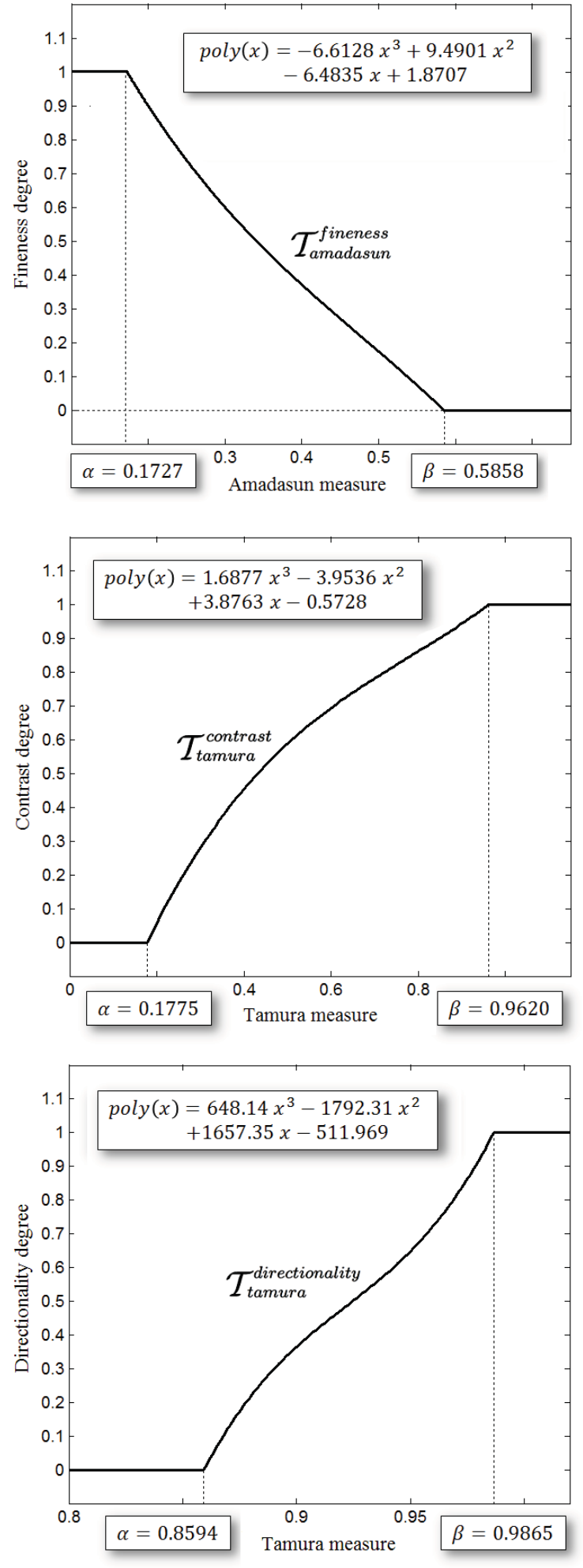

Figure 2: Proposed membership function $\mathcal{T}_{k}^{p}$ corresponding to fineness, contrast and directionality properties. 
(a)
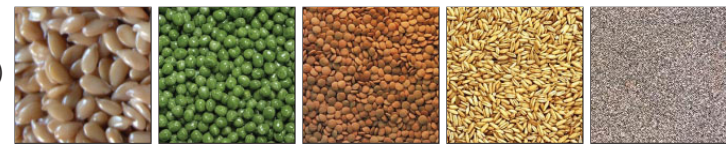

(b)
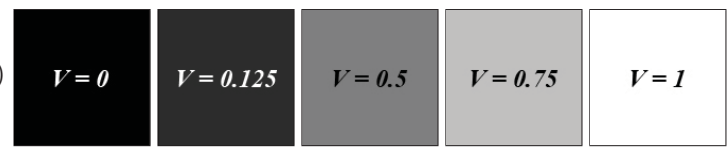

(c)
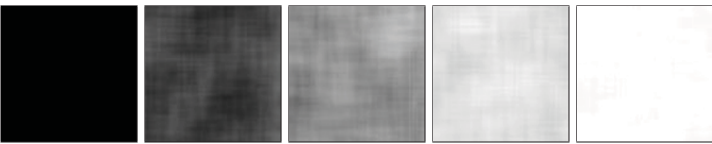

Figure 3: Result for a collection of texture images (a), showing the human assessments about the presence degree of fineness (b), and the membership degrees obtained by applying the fineness model (c).

$F_{k}^{p} \in \mathcal{F}^{p}$ for the properties of fineness, contrast and directionality, respectively. Note that this value can be viewed as the goodness of each measure to represent the perception of the corresponding property. These tables have been sorted in increasing order of the fitting errors.

In addition, the test error for each measure has been calculated by using the subset of points $\Psi_{\text {test }}^{p, k}$ and it is shown in the third column of these tables. In our approach, this error is calculated as the mean absolute difference between the values $v_{i}^{p}$ and the degrees obtained by applying the function $\mathcal{T}_{k}^{p}$ to the values $m_{i, w}^{p, k}$, for all the points $\left(m_{i, w}^{p, k}, v_{i}^{p}\right) \in \Psi_{\text {test }}^{p, k}$, i.e.

$$
E^{\text {test }}=\frac{\sum_{\left(m_{i, w}^{p, k}, v_{i}^{p}\right) \in \Psi_{t e s t}^{p, k}}\left|\mathcal{T}_{k}^{p}\left(m_{i, w}^{p, k}\right)-v_{i}^{p}\right|}{\operatorname{card}\left(\Psi_{\text {test }}^{p, k}\right)}
$$

with $\operatorname{card}\left(\Psi_{\text {test }}^{p, k}\right)$ being the cardinality of $\Psi_{\text {test }}^{p, k}$.

In our experiments, the membership functions with the lowest error are obtained by using the measure of Amadasun in the case of fineness and the measures of Tamura in the case of contrast and directionality. Figure 2 shows the parameters and the graphical representation of these membership functions.

\section{Results}

In this section, the fuzzy models proposed for fineness, contrast and directionality properties will be applied to several examples in order to analyze its performance. In particular, the membership functions $\mathcal{T}_{k}^{p}$ with least fitting error and least test error (shown in Figure 2) will be used.

For the first experiment, we have considered the collection of texture images shown in Figure 3(a), each one with a different increasing perception degree of fineness. These images are part of the set $\mathcal{I}^{\text {fineness }}$ used in the poll, so human assessments about fineness presence are available in order to compare with the obtained results. (a)


(b)
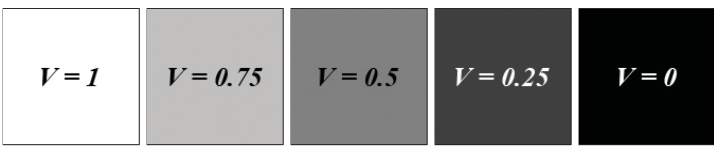

(c)
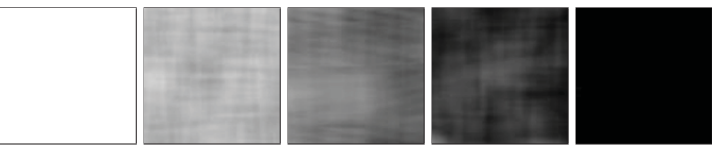

Figure 4: Result for a collection of texture images (a), showing the human assessments about the presence degree of contrast (b), and the membership degrees obtained by applying the contrast model (c).

(a)
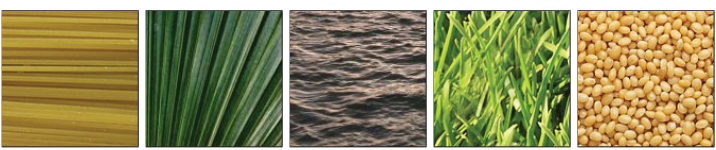

(b)
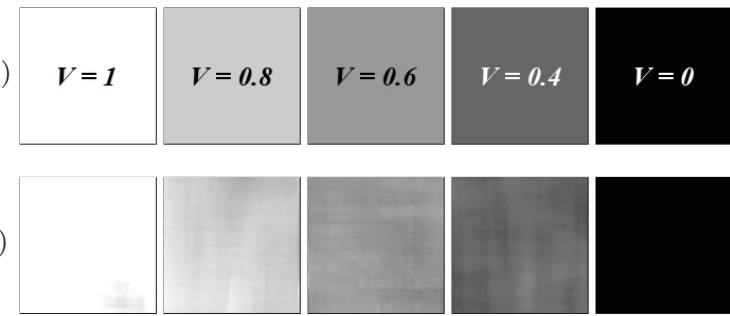

Figure 5: Result for a collection of texture images (a), showing the human assessments about the presence degree of directionality (b), and the membership degrees obtained by applying the directionality model (c).

Figure 3(b) shows an ideal mapping from the original texture images to their fineness values, where all pixels corresponding to the same texture image have been mapped using the human assessment associated to that image. These assessments (between 0 and 1) have been mapped into a gray level from 0 to 255 , so that a white pixel in the mapping indicates maximum perception of fineness, while a black one indicates no perception of fineness (maximum perception of coarseness).

Figure 3(c) shows a mapping from the original texture images to their fineness values obtained by applying the proposed model. For each pixel in the original images, a centered window of size $32 \times 32$ has been analyzed and its fineness membership degree has been calculated. This degree has been mapped into a gray level from 0 to 255 . It can be noticed that our model captures the evolution of the perception degrees of fineness, and the obtained mapping, that represents the estimated presence degree of this property, can be directly interpreted by humans. 


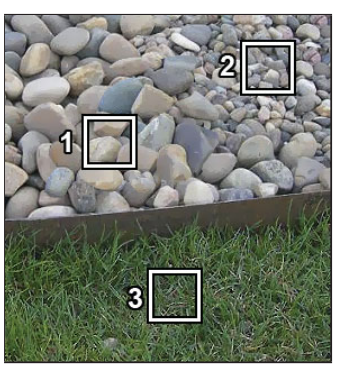

(a)

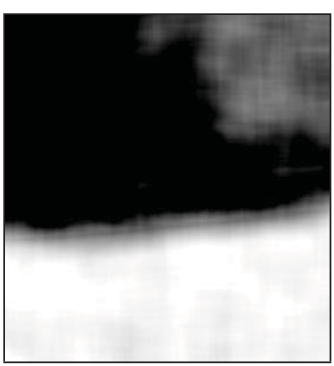

(b)

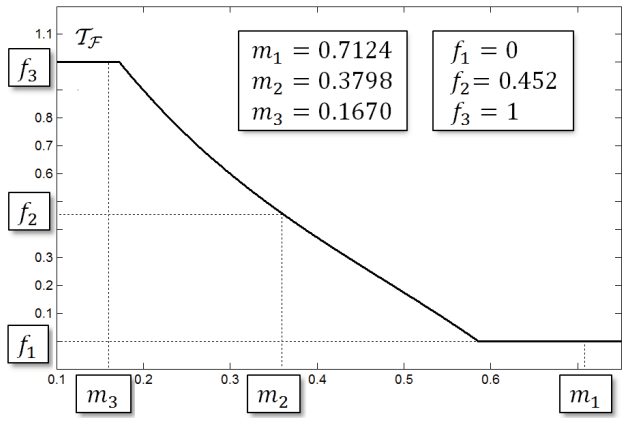

(c)

Figure 6: Result for a natural image. (a) Original image. (b) Mapping from the original image to its fineness values using the proposed model. (c) Graphical representation of the proposed model and calculation detail for the three windows shown in the original image.

Two similar experiments, shown in figures 4 and 5 , have been performed for the properties of contrast and directionality. It can be noticed that the results obtained with our models (figures 4(c) and 5(c)) match what a human would expect, capturing the evolution of the perception degrees of contrast and directionality, respectively.

For the next experiment, let's consider Figure 6(a), corresponding to a natural image where several textures with different perception degrees of fineness are shown. Figure 6(b) shows a mapping from the original image to its fineness values using the proposed model. It can be noticed that three different degrees of fineness are shown: a coarse texture (pixels in black) corresponding to the big stones, a fine texture (pixels in white) corresponding to the grass, and an intermediate coarseness texture (pixels with an intermediate gray level) corresponding to gravel. As example, Figure 6(c) shows a graphical representation of the mapping process for three different windows of the original image (marked as 1, 2 and 3 in Figure 6(a)). As in the previous experiments, it can be noticed that the proposed model captures the evolution of the perception degrees of fineness, obtaining results that match what a human would expect. Two similar experiments, shown in figures 7 and 8 , have been performed for the properties of contrast and directionality, obtaining mappings that can be directly interpretable by humans.

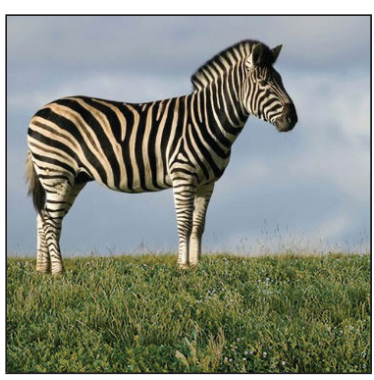

(a)

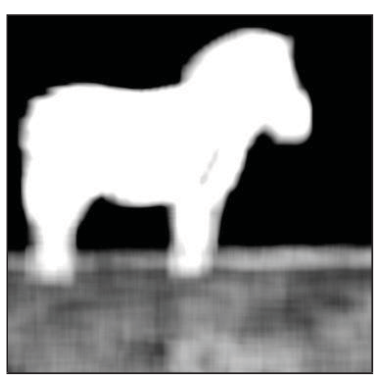

(b)
Figure 7: Result for a natural image. (a) Original image. (b) Mapping from the original image to its contrast values using the proposed model.

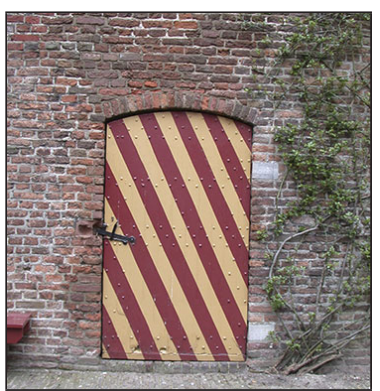

(a)

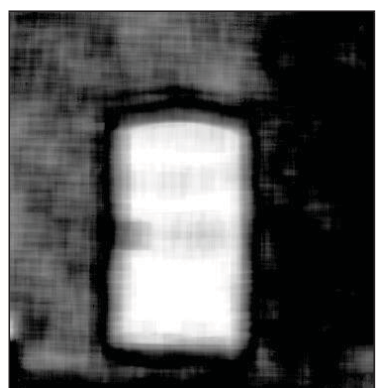

(b)
Figure 8: Result for a natural image. (a) Original image. (b) Mapping from the original image to its directionality values using the proposed model.

\section{Conclusions}

In this paper, a perception-based fuzzy approach for texture modelling has been proposed. In this approach, texture properties have been modelled by means of fuzzy sets defined on the domain of computational measures of the corresponding property. In order to define these models, parametric functions have been employed, where the corresponding parameters have been calculated by taking into account the relationship between the computational measures and the human perception of the property. This way, the shape of the membership function has been adjusted to represent this relationship, and the obtained membership degrees match what a human would expect. We have concluded that the models obtained by using the measure of Amadasun in the case of fineness, and the measures of Tamura for contrast and directionality, have the best ability to represent the perception of the corresponding property. Moreover, the use of a unique fuzzy set to model the texture contrast as a whole has allowed its application to pattern recognition problems (e.g. the examples shown in the previous section).

\section{References}

[1] A.R. Rao and G.L. Lohse. Identifying high level features of texture perception. Graphical 
Models and Image Processing, 55(3):218-233, 1993.

[2] H. Tamura, S. Mori, and T. Yamawaki. Textural features corresponding to visual perception. IEEE Trans. on Systems, Man and Cybernetics, 8:460-473, 1978.

[3] S. Battiato, G. Gallo, and S. Nicotra. Perceptive visual texture classification and retrieval. In Proceedings of International Conference on Image Analysis and Processing, pages 524-529, 2003.

[4] B. S. Manjunath. Introduction to MPEG7, Multimedia Content Description Interface. John Wiley and Sons, Ltd., New York, Jun 2002.

[5] M. Nirmala, K. Karthikeyan, S. Appalabatla, and R.A. Ahmed. Image interpretation based on similarity measures of visual content descriptors. An insight. International Journal of Computer Science and Emerging Technologies, 2(2):242-248, 2011.

[6] H. Aboulmagd, N. El-Gayar, and H. Onsi. A new approach in content-based image retrieval using fuzzy. Telecommunication Systems, 40(1):55-66, 2008.

[7] M. Flicker, H. Sawhney, W. Niblack, J. Ashley, Q. Huang, B. Dom, M. Gorkani, J. Hafner, D. Lee, D. Petkovic, D. Steele, and P. Yanker. Query by image and video content: the QBIC system. IEEE Computer, 28(9):23-32, 1995.

[8] H.C. Lin, C.Y. Chiu, and S.N. Yang. Finding textures by textual descriptions, visual examples, and relevance feedbacks. Pattern Recognition Letters, 24(14):2255-2267, 2003.

[9] R.M. Haralick. Statistical and structural approaches to texture. Proceedings IEEE, 67(5):786-804, 1979.

[10] M. Amadasun and R. King. Textural features corresponding to textural properties. IEEE Transactions on Systems, Man and Cybernetics, 19(5):1264-1274, 1989.

[11] K.I. Chang, K.W. Bowyer, and M. Sivagurunath. Evaluation of texture segmentation algorithms. In Proc. IEEE Computer Society Conference on Computer Vision and Pattern Recognition, volume 1, pages 294-299, 1999.

[12] Todd R. Reed and J.M. Hans Du Buf. A review of recent texture segmentation and feature extraction techniques. CVGIP: Image Understanding, 57(3):359-372, 1993.

[13] S. Kulkarni and B. Verma. Fuzzy logic based texture queries for CBIR. In Proc. 5th International Conference on Computational Intelligence and Multimedia Applications, pages 223228, 2003.

[14] B. Verma and S. Kulkarni. A fuzzy-neural approach for interpretation and fusion of colour and texture features for CBIR systems. Applied Soft Computing, 5(1):119-130, 2004.

[15] W.T. Lin, C.H. Lin, T.H. Wu, , and Y.K.
Chan. Image segmentation using the k-means algorithm for texture features. In Proc. International Conference on Computer, Electrical, and Systems Science, and Engineering (ICCESSE), pages 26-28, 2010.

[16] W. Niblack, R. Barber, W. Equitz, M. Flickner, E.H. Glasman, D. Petkovic, P. Yanker, C. Faloutsos, and G. Taubin. The QBIC project: Querying images by content, using color, texture, and shape. In Proceedings of Storage and Retrieval for Image and Video Databases (SPIE), pages 173-187, 1993.

[17] P. Brodatz. Textures: a photographic album for artists and designers. Dover Publishing Co., New York, 1966.

[18] R.R. Yager. On ordered weighted averaging aggregation operators in multicriteria decisionmaking. IEEE Trans. on SMC, 18(1):183-190, 1988.

[19] N. Abbadeni, N. Ziou, and D.S. Wang. Autocovariance-based perceptual textural features corresponding to human visual perception. In Proc. of 15th International Conference on Pattern Recognition, volume 3, pages 901904, 2000.

[20] S. Peleg, J. Naor, R. Hartley, and D. Avnir. Multiple resolution texture analysis and classification. IEEE Transactions on Pattern Analysis and Machine Intelligence, (4):518-523, 1984.

[21] J. Canny. A computational approach to edge detection. IEEE Transactions on Pattern Analysis and Machine Intelligence, 8(6):679698, 1986.

[22] S.I. Kim, K.C. Choi, and D.S. Lee. Texture classification using run difference matrix. In Proc. of IEEE 1991 Ultrasonics Symposium, volume 2, pages 1097-1100, December 1991.

[23] J.S. Weszka, C.R. Dyer, and A. Rosenfeld. A comparative study of texture measures for terrain classification. IEEE Trans. on SMC, 6:269-285, 1976.

[24] C. Sun and W.G. Wee. Neighboring gray level dependence matrix for texture classification. Computer Vision, Graphics and Image Processing, 23:341-352, 1983.

[25] M.M. Galloway. Texture analysis using gray level run lengths. Computer Graphics and Image Processing, 4:172-179, 1975.

[26] S.D. Newsam and C. Kammath. Retrieval using texture features in high resolution multispectral satellite imagery. In SPIE conference on Data Mining and Knowledge Discovery: Theory, Tools, and Technology VI, volume 5433, pages 21-32, April 2004.

[27] A.E. Beaton and J.W. Tukey. The fitting of power series, meaning polynomials, illustrated on band-spectroscopic data. Technometrics, 16:147-185, 1974. 